\title{
Effect of Post-processing on Photoluminescence and Surface Composition of Porous Silicon
}

\author{
Xi-Feng ZHANG ${ }^{a,{ }^{*}}$, Han ZHAO ${ }^{b}$, Hong-Xia DONG ${ }^{c}$, Yu WANG ${ }^{d}$, Kun FAN ${ }^{e}$ \\ School of Chemistry and Chemical Engineering, Jiangsu University, Zhenjiang 212013, PR China \\ azhangxf_chzh@163.com, b1607399699@qq.com, '841759188@qq.com, d2281012609@qq.com, \\ eFankun19941010@163.com \\ ${ }^{*}$ Corresponding author
}

Keywords: Silicon, Photolumimescence, Chemical Analysis, Electrochemical Processes, X-ray Spectroscopy, Surface Treatment.

\begin{abstract}
Porous silicon (PS) with $6.13 \mathrm{~nm}$ average pore diameter, 20.6 $\mu \mathrm{m}$ thickness layer and $70.8 \%$ porosity prepared by galvanostatic electrochemical anodisation of p-type silicon wafer in aqueous hydrofluoric acid (HF)/ethanol electrolyte. The freshly prepared PS was processed by time effect, nitric acid treatment, cathodic reduction, and hydrogen peroxide treatment, respectively. Samples were characterized and analyzed by field emission scanning electron microscopy (FESEM), Photolumimescence (PL) and X-ray photoelectron spectroscopy (XPS) before and after post-processing. The PL spectra showed that PS had three PL peaks near to $425 \mathrm{~nm}, 486 \mathrm{~nm}$ and $530 \mathrm{~nm}$, respectively, the strongest luminous intensity at $425 \mathrm{~nm}$ obtained when PS followed $6 \mathrm{~h}$ in hydrogen peroxide post-processing, and the difference effect on PL via various post-processing were also discussed. From XPS spectra analysis, the surface composition of PS via different post-processing method was carried out that the oxygen mainly existed in the form of Si-O.
\end{abstract}

\section{Introduction}

Porous silicon (PS) has attracted a lot of interests after the discovery of its efficient visible room temperature photoluminescence (PL) [1]. It was applied as new functional materials, because of its unique chemical and physical properties, such as direct and wide modulated energy band gap, high resistivity, vast surface area-to-volume ratio, electronic, optical, mechanical and thermal properties etc [2-7]. Therefore, PS has been researched in comprehensive fields and far from being complete. Furthermore, due to its large internal surface cover and optical properties, the biological properties and possible applications of PS have been intensively studied by many groups [8-12].

However, there are potential problems in the application of PS. The luminous intensity and efficiency of PS are significantly decreased after a period of storage because of its instability when it is used as a light emitting material, or its surface covered with relatively small holes prompts the reduction of the efficiency adsorption and sensitivity of PS which applied as a sensor. Therefore, in order to solve these problems, PS should be post-processing, such as surface oxidation [13, 14], surface passivation [15-18], ion implantation [19], organic compound [20] and solution erosion [21], and many others $[22,23]$.

In this article, we study the prepared PS which was carried out following post-processing method as time effect, nitric acid treatment, cathodic reduction, and hydrogen peroxide treatment, respectively. The main objective of this study is to enhance the intensity and stability of the PL of PS. The best condition for the post-processing of PS has been defined and discussed. In addition, in order to study the effect of the surface composition of PS after post-processing, X-ray photoelectron spectroscopy (XPS) is used to study the nature of the chemical bonding before and after post-processing.

\section{Material, Methods and Experimental}

\section{Porous silicon Fabrication and Morphology Characterization}

The PS was fabricated by electrochemical etching as reference [9] reported, with some modified by ourselves. All experiments were conducted on square samples $(10 \mathrm{~mm} \times 10 \mathrm{~mm})$ cut from a 
boron-doped p-type (100) silicon wafers with a resistivity of $0.001-0.005 \Omega \cdot \mathrm{cm}$, thickness of 500 $\mu \mathrm{m}$ bought from ZHEJIANG LEAJING Crystal Silicon materials CO. LTD.. Prior to synthesis, the silicon wafers were ultrasonically cleaned with deionized water, acetone, and ethanol for 20 min in order to remove the organic contaminants. Afterward, the silicon wafers were immersed in $5 \mathrm{wt} \%$ aqueous hydrofluoric acid (HF) for $30 \mathrm{~s}$ to remove the native oxide. The growing of the porous layers was provided by the anodization in an electrolyte consisting of $1: 1(\mathrm{v}: \mathrm{v})$ ethanol and aqueous 40 wt. \% hydrofluoric acid in a typically constructed Teflon cell, under 30min etching time and $30 \mathrm{~mA} / \mathrm{cm}^{2}$ corrosion current density. After corrosion, PS was cleaned 2 times in the dichloromethane by ultrasonic cleaner, each time for $20 \mathrm{~min}$. Then, PS was dried in a vacuum drying oven at room temperature, and stored for use. PS morphology was investigated by Model S-4800 field emission scanning electron microscope (Japan, Hitachi High-Technologies Corporation), and its porosity was calculated by the weightlessness method.

\section{Porous silicon Post-processing and Photoluminescence Characterization}

Four methods of post-processing of PS as time effect, nitric acid treatment, cathodic reduction and hydrogen peroxide treatment were used [24]. The fresh PS without any post-processing marked as P0. In the aging treatment, the PL intensity of the sample P0 was characterized in fresh preparation and placed at room temperature for 7, 14, 30, 60 and 120 days, respectively. In the nitric acid treatment, the solution contained a mixture of nitric acid (69 wt. \%), deionized water with the volume ratio of 1:2, 1:4, 1:6 and 1:8 were configured respectively. Then the samples marked X1, $\mathrm{X} 2, \mathrm{X} 3$ and $\mathrm{X} 4$ respectively were placed in the different concentrations of the nitric acid solution and soaked for 2 hours. In the cathodic reduction process, after normal etching time, the samples were prolonged theirs etching time after the anode and cathode were swapped, and the corrosion current density was also changed as $10 \mathrm{~mA} / \mathrm{cm}^{2}$. The samples with prolongation etching time for 5 min, $10 \mathrm{~min}, 15 \mathrm{~min}, 20 \mathrm{~min}$ were labeled as Y1, Y2, Y3 and Y4, respectively. In the hydrogen peroxide process, the flesh samples immersed into hydrogen peroxide ( $\mathrm{H} 2 \mathrm{O} 2,40 \mathrm{wt} . \%)$ for $2 \mathrm{~h}, 4 \mathrm{~h}$, $6 \mathrm{~h}$, and $8 \mathrm{~h}$ respectively were named as H1, H2, H3 and H4. PL spectra of above samples were detected at room temperature using a Cary Eclipse Fluorescence spectrometer (America, Varian) with an argon ion laser (370 $\mathrm{nm}$ line) for excitation.

\section{Analysis of Surface Composition of PS before and after Post-processing}

In this section, sample P0, sample Y2 and H3 were characterized by XPS with a PHI5300 spectrometer using $\mathrm{Al} \mathrm{K \alpha}(12.5 \mathrm{KV}) \mathrm{X}$-ray source.

\section{Results and Discussion}

\section{Morphology of PS}

The morphology of freshly prepared PS before and after hydrogen peroxide treatment is confirmed by FESEM observations (Fig.1). It is revealed that the structure of PS sample contains uniform, nano-size circular-pores $(6.13 \mathrm{~nm}$ average pore diameter calculated via Nano Measurer 1.2 Particle Size Analysis Software, 20.6 $\mu \mathrm{m}$ thickness layer shown in Fig 1(b)) in vertical direction, and increasing pore sizes with the growth of etching time and depth (Fig 1(c)). The pores have a vertical direction trend that refers to the effect of electric field force limited holes transferring in the vertical direction rather than moving randomly, which named the priority of holes on reaching into tip reaction. However, with the increase of corrosion time and degree, some holes are not exhausted and remain on the hole-wall to stimulate lateral corrosion which cannot be ignored and result the pore size increasing with the depth of the porous silicon hole-wall.

With the weightlessness method [10], the porosity of PS is calculated as $70.8 \%$. The detail of weightlessness method is summarized in the following equation: $\varepsilon=\frac{V_{\mathrm{P}}}{V_{\mathrm{Si}}}=\frac{V_{\mathrm{P}} \rho_{\mathrm{Si}}}{V_{\mathrm{Si}} \rho_{\mathrm{Si}}}=\frac{m_{1}-m_{2}}{m_{1}-m_{3}}$

In the above equation, $\varepsilon, V_{\mathrm{p}}$ and $V_{\mathrm{Si}}$ denote porosity, hole volume, total volume of PS, respectively. $\rho_{\mathrm{Si}}$ is represented as the density of monocrystalline silicon wafer. $m_{1}$ and $m_{2}$ indicate the mass of silicon wafer before and after etching. $m_{3}$ is identified as the mass of etched silicon wafer after removed PS layer with $1 \mathrm{~mol} / \mathrm{L} \mathrm{NaOH}$ solution and vacuum drying. 


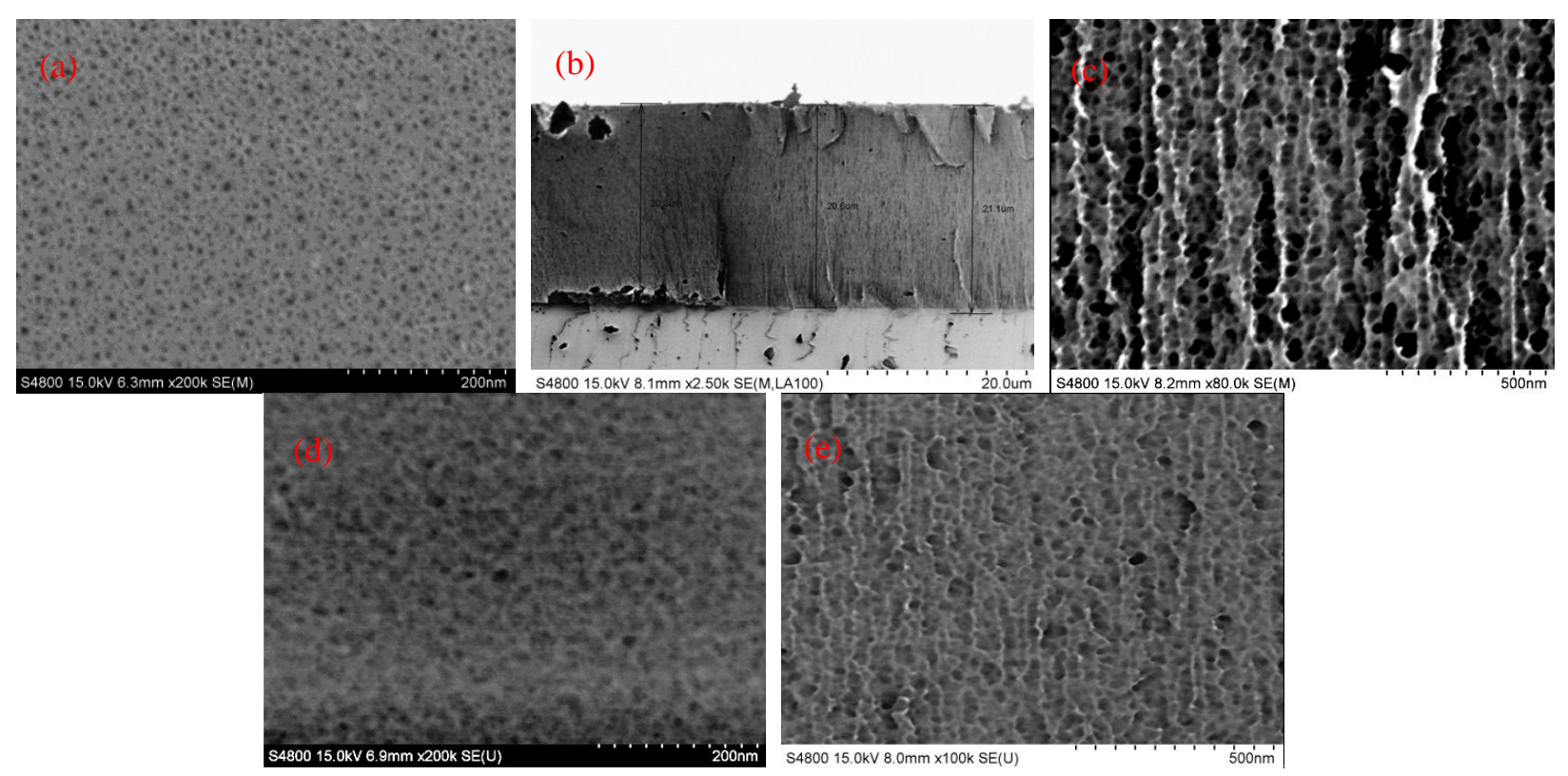

Fig.1 Planar view (a,d) and Cross-sectional view (b,c,e) FESEM images of fresh porous silicon before and after hydrogen peroxide treatment

\section{Effects of Post Treatments on the Properties of PS Photoluminescence}

The analysis of aging time, nitric acid treatment, cathodic reduction, and hydrogen peroxide effect on the PL properties of PS has been established by the spectra of above samples in Fig.2. The PL spectra indicate that there are three PS emission peaks, and the main peak is at about $425 \mathrm{~nm}$, while the weak PL peaks appear at around $486 \mathrm{~nm}$ and $530 \mathrm{~nm}$. From Fig.2, the cathodic reduction and hydrogen peroxide treatment have the greater influence on the PL of PS. The details have shown as follow.

With the increasing of the aging time effect, the intensity of PL peak at $425 \mathrm{~nm}$ has been enhanced with minuscule changes at $486 \mathrm{~nm}$ and $530 \mathrm{~nm}$, with increasing blue shifts at $530 \mathrm{~nm}$ which is identical with literature reference [1] (Fig.2 a). It can be deduced from the silicon band gap width. The bulk silicon band gap width is $1.14 \mathrm{eV}$, which is lower than red light emitting peak position in the PL spectrum energy. However, the nanometer size effect derived from PS samples (Fig.1) makes it increase effective forbidden band width. Therefore, PS light emitting peak at around $530 \mathrm{~nm}$ should be caused by the quantum confinement effect [25]. In process of aging time, the nanometer size of PS appears little differentiation (FESEM images no), which also explains why at $530 \mathrm{~nm}$ PL peak has little change in Fig. 2 a. At the same time, the peak intensity at 486nm is tiny change, which would be explained by the surface state model [26]. As aging time goes on, the intensity of $425 \mathrm{~nm}$ has certain enhancement, which may be due to the increasing of the $\mathrm{SiO}_{2}$ content on PS surface when it is saved in the air, and then the light emission center should be located in the oxygen vacancy $\mathrm{SiO}_{2}$ defects, and the emission center increases as the growth of aging time. Therefore, we speculate that the peak at $425 \mathrm{~nm}$ is supposed to be caused by defect in PS, which can be described by the defect model [27].

It can be obviously observed that the PL intensities of all the samples with the nitric acid treatment have almost the same values in Fig. 2 b, while as the peak near $425 \mathrm{~nm}$ of X1 have a little enhancement, which may be included as following. The concentration of nitric acid of X1 is larger than that of other samples, and leads up to additional corrosivity in the $\mathrm{X} 1$ to make its surface $\mathrm{SiO}_{2}$ content increased slightly.

From Fig. 2 c, there are varying degrees of increase intensity of these three PL peaks and slight blue shifts at $486 \mathrm{~nm}$ and $530 \mathrm{~nm}$. In the cathodic reduction process, the increase of the peak's intensity at $530 \mathrm{~nm}$ due to the change of the aperture of the PS increases the quantum confinement effect, and the enhancement of the peak at $486 \mathrm{~nm}$ belong to the change of the PS surface. The maximum changed intensity appeared at $425 \mathrm{~nm}$ might come from the enhancement of the defect role with more and more $\mathrm{Si}-\mathrm{O}$ defects emergence during the cathodic reduction process. From Fig.4 we also find that the luminous intensity of peak at $425 \mathrm{~nm}$ of $\mathrm{Y} 2$ is the strongest. This can be 
conclude that the defects on the surface of PS is increase during the first period reduction time, and are partially reacted after more than 10 min reduction time, which also lead the decrease of the luminous intensities of Y3 and Y4 compared with Y2.

The hydrogen peroxide effect on the properties of PS PL is shown in Fig.2 d. The reasons of hydrogen peroxide treatment affects on the intensity of PS PL peak at 530nm and 486nm are the same as the reasons of the cathode reduction treatment does (the nanometer size of PS appears bigger after hydrogen peroxide treatment seen in Fig 1(d, e)). The change of the peak intensity at $425 \mathrm{~nm}$ might produce the $\mathrm{Si}-\mathrm{H}$ in addition to the $\mathrm{Si}-\mathrm{O}$ defects. This is because hydrogen peroxide contains $-\mathrm{OH}$ bond, which is helpful in strengthen the PL of PS. From Fig. 2 d, we also clearly find that the luminous of $\mathrm{H} 3$ at $425 \mathrm{~nm}$ peak has the strongest intensity which belongs to the increase of $\mathrm{Si}-\mathrm{H}$ and $-\mathrm{OH}$ on PS surface with the extension of treatment time. It can be concluded that the $6 \mathrm{~h}$ processing time is the optimum choice to enhance the PL of PS.
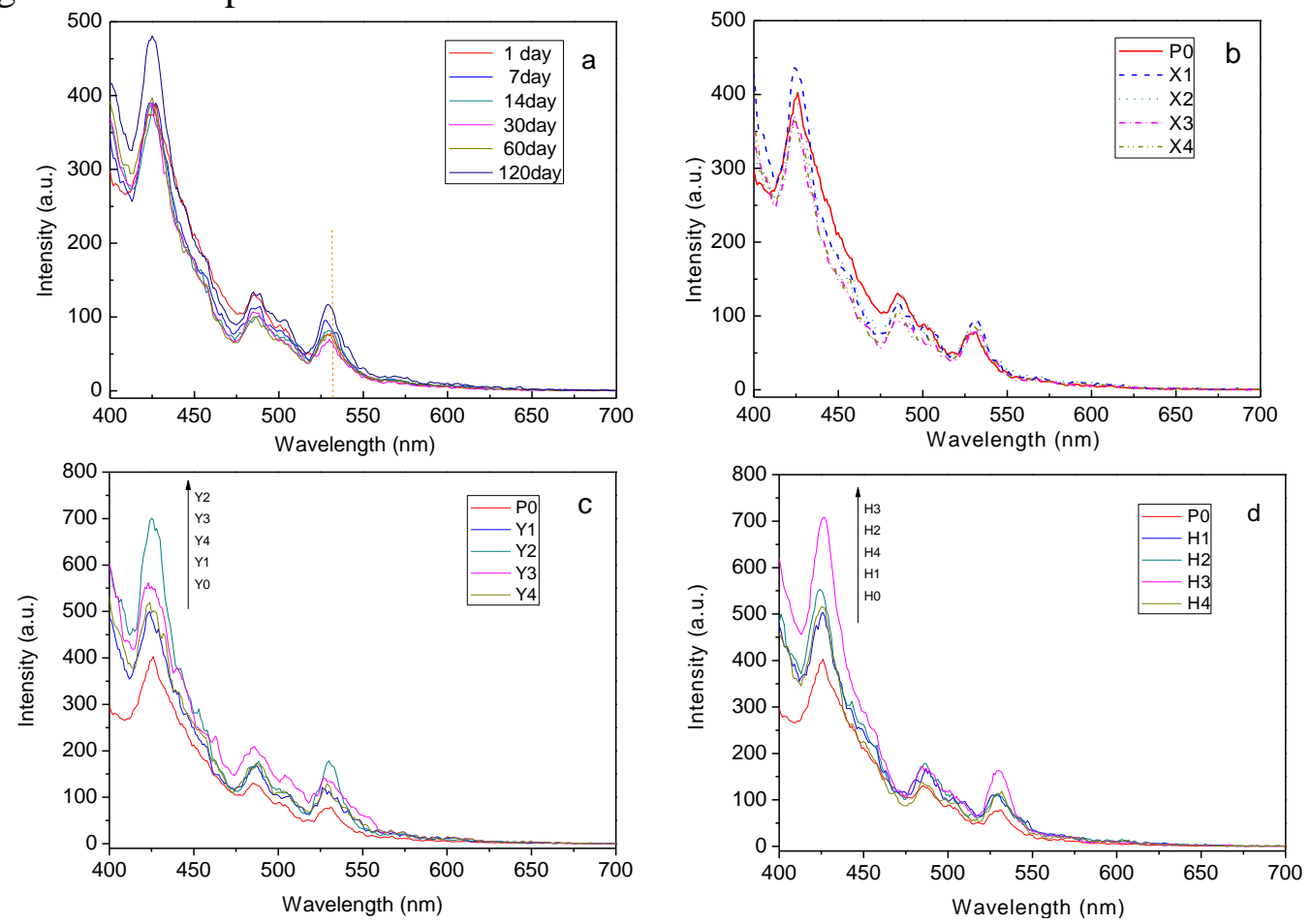

a: Aging time; b: Nitric acid treatment(X1 1:2, X2 1:4, X3 1:6, X4 1:8 (v:v, nitric acid 69 wt\% than deionized water)); c: Cathodic reduction(Y1 5min, Y2 10min, Y3 15min, Y4 20min); d: Hydrogen peroxide(H1 2h, H2 4h, H3 6h, H4 8h)(P0 as the blank sample)

Fig. 2 Effect of post-processing on the properties of PS photoluminescence

\section{Analysis of Surface Composition of PS}

The XPS spectra to analyze the surface composition of PS before and after post-processing are shown in Fig. 6 and Fig.7. The spectra of P0, Y2 and H3 are nearly the same curves except the disappearance of $\mathrm{Cu}$ element in the sample $\mathrm{H} 3$, which is the same as the results shown in Tab.1. The sources of the $\mathrm{Cu}$ element should come from $\mathrm{Cu}$ back contact which transfer into PS pores via ions accompanied with the remove of electron hole during etching process. Followed with hydrogen peroxide post-processing, $\mathrm{Cu}$ dissolve in solution, which can be used to explain why there is no $\mathrm{Cu}$ element in the XPS spectra of $\mathrm{H} 3$.

Tab.1 The surface composition of the samples P0, Y2 and H3, respectively

\begin{tabular}{cccccc}
\hline Sample & $\begin{array}{c}\text { C1s } \\
(\%)\end{array}$ & $\begin{array}{c}\text { O1s } \\
(\%)\end{array}$ & $\begin{array}{c}\text { F1s } \\
(\%)\end{array}$ & $\begin{array}{c}\text { Si2p } \\
(\%)\end{array}$ & $\begin{array}{c}\text { Cu2p } \\
(\%)\end{array}$ \\
\hline P0 & 32.51 & 18.35 & 3.64 & 44.85 & 0.64 \\
Y2 & 25.20 & 31.80 & 2.89 & 39.77 & 0.35 \\
H3 & 15.04 & 51.60 & 1.05 & 32.31 & 0 \\
\hline
\end{tabular}


In addition, we find the $\mathrm{O} 1 \mathrm{~s}$ peak in the $\mathrm{H} 3$ significantly enhances while $\mathrm{Y} 2$ shows soften, which may put down to hydrogen peroxide treatment made the $\mathrm{O}$ elements of $\mathrm{H} 3$ surface increase to increase PS surface defects. Fig.7 shows the XPS spectra after fitting of P0's Si and Cu. From Fig.7a we can find the $\mathrm{Si}$ is mainly in the form of $\mathrm{Si}-\mathrm{Si}$ and $\mathrm{Si}-\mathrm{O}$. From Fig.7b we can find the $\mathrm{Cu}$ only exists in the form of $\mathrm{CuO}$, but it is fewer.

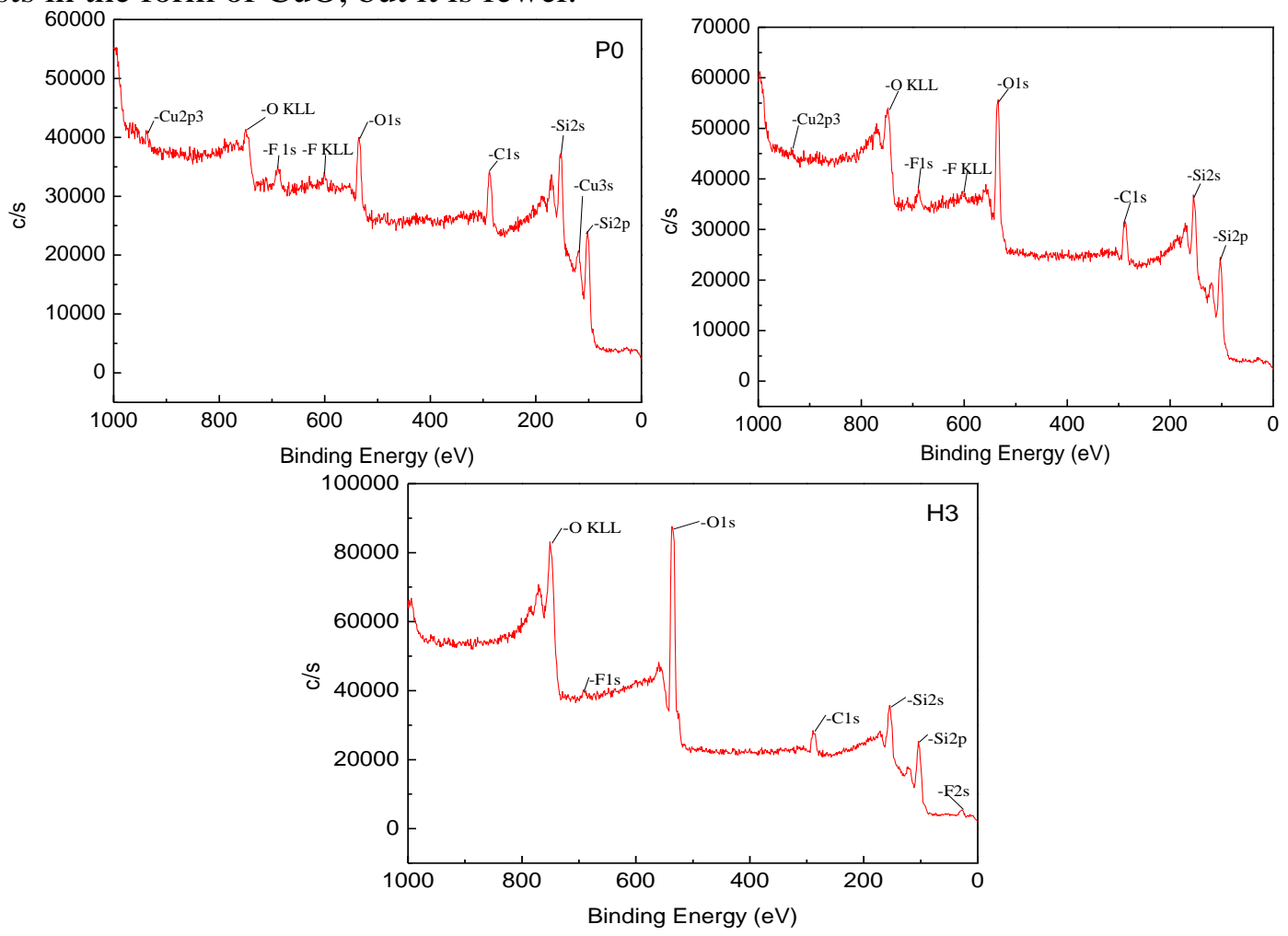

Fig.6 XPS spectrum of the surface of the samples P0, Y2 and H3
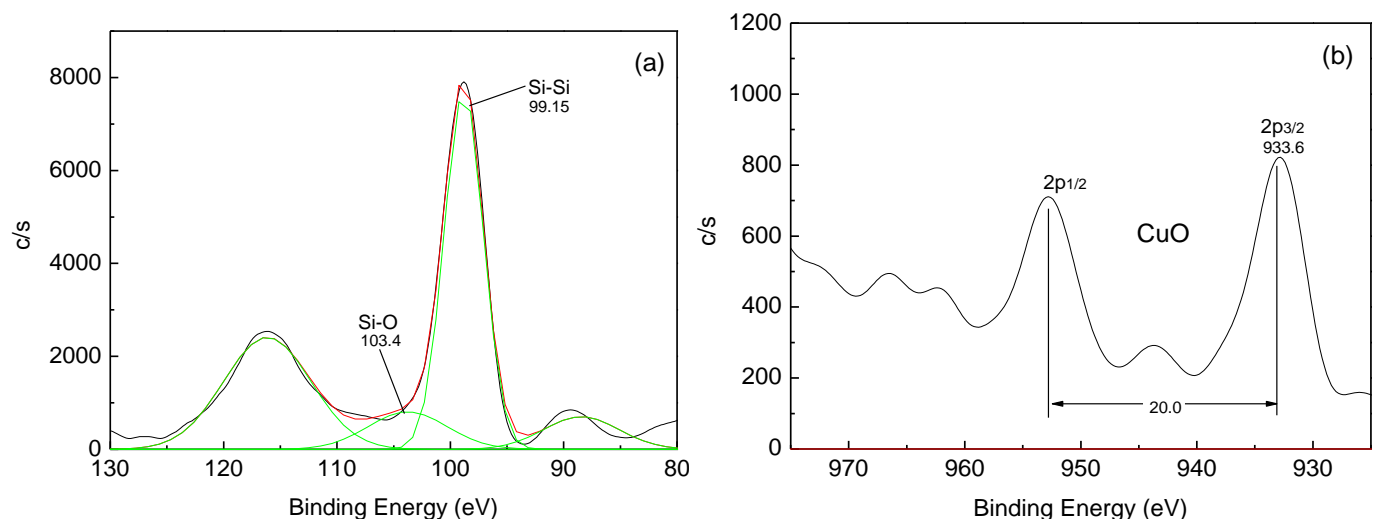

Fig.7 XPS spectrum of the Si and $\mathrm{Cu}$ of the sample P0, (a) $\mathrm{Si}$, (b) $\mathrm{Cu}$

\section{Summary}

On the basis of this research, the following conclusions can be drawn. Porous silicon (PS) with 6.13 $\mathrm{nm}$ average pore diameter, $20.6 \mu \mathrm{m}$ thickness layer and $70.83 \%$ porosity is prepared by anodic electrochemical corrosion. PS have three PL peaks near to $425 \mathrm{~nm}, 486 \mathrm{~nm}$ and $530 \mathrm{~nm}$, respectively. The PL of PS is determined by the quantum confinement effect, the surface states and the defects result of joint action, and the defects play a major role. In the post-processing, the cathodic reduction and hydrogen peroxide treatment have the greatest influence on the PL of PS. When PS processed $6 \mathrm{~h}$ in hydrogen peroxide, the luminous intensity of PS is the strongest. In the surface of PS, the oxygen mainly exists in the form of Si-O. 


\section{References}

[1]L. T. Canham, Applied Physics Letters 57 (1990) 1046-1048.

[2]A. G. Cullis, L. T. Canham, P. D. J. Calcott, Journal of Applied Physics, 82(1997) 909-965

[3]A. Ensafi Ali, M. Mokhtari Abarghoui, B. Rezaei, Electrochimica Acta, 123(2014) 219-226.

[4]N. Naderi, M.R. Hashim, T.S.T. Amran, Superlattices and Microstructures 51 (2012) 626-634.

[5]H. Dirk, B. Vasani Roshan, J. P. McInnes Steven, V. Ellis Amanda, H. Voelcker Nicolas, ACS Macro Letters 1 (2012) 919-921.

[6]J. Kinnari, Päivi, L. K. Hyvönen Maija, M. Mäkilä Ermei, M. H. Kaasalainen, A. Rivinoja, J. J. Salonen, J. T. Hirvonen, P. M. Laakkonen, H. A. Santos, Biomaterials, 34 (2013) 9134-9141.

[7]A. Kermad, S. Sam, N. Ghellai, K. Khaldi, N. Gabouze, Materials Science and Engineering B 178 (2013) 1159-1164.

[8]R. E. Serda, A. Mack, M. Pulikkathara, A. M. Zaske, C. Chiappini, J. R. Fakhoury, D. Webb, B. Godin, J. L Conyers, X. W. Liu, J. A. Bankson, M. Ferrari, Small 6 (2010) 1329-1340.

[9]J. H. Park, L. Gu, G. V Maltzahn, E. Ruoslahti, S. N. Bhatia, M. J. Sailor, Nature Materials 8 (2009) 331-336.

[10]M.J. Sailor, Porous Silicon in Practice: Preparation, Characterization and Applica- tions, Wiley-VCH Verlag GmbH and Co.KGaA,Winheim Germany, 2012.

[11]S. W. Lee, S. Kim, J. Malm, O. C. Jeong, H. Lilja, T. Laurell, Analytica Chimica Acta 796 (2013) 108-114.

[12]R. Shabannia, H. Abu Hassan, Superlattices and Microstructures 62 (2013) 242-250.

[13]B. M. Kostishko, I. P. Puzov, Yu. S. Nagornov, Technical Physics Letters 26 (2000) 26-28.

[14]Y. S. Zhang, Z. M. Yang, D. L. Liu, E. Y Nie, X. Bai, Z. W. Li, H. B. Song, Y. Zhou, W. Y. Li, M. Gong, X. S. Sun. Journal of Luminescence, 130 (2010) 1005-1010.

[15]X. W. Du, Y. W. Lu, J. P. Liu, J. Sun, Applied Surface Science 252 (2006) 4161-4166.

[16]Y. H. Zhang, X. J. Li, L. Zheng, Q. W. Chen, Physical Review Letters 81 (1998) 1710.

[17]C. H. Chen, Y. F. Chen, Applied Physics Letters 75 (1999) 2560-2562.

[18]D. L. Zhu, Q. W. Chen, Y. H, Modem Physics. Letters B 15 (2002) 1077-1085.

[19]M. Rahmani, A. Moadhen, M. A. Zaïbi, A. Lusson, H. Elhouichet, M. Oueslati. Journal of Alloys and Compounds, 485 (2009) 422-426.

[20]N. P. Mandal, A. Sharma, S. C. Agarwal, Journal of Applied Physics 100 (2006) 4308-4311.

[21]A. G Rozhin, N. I Klyui, V. G. Litovchenko, Yu. P Piryatinskii, Materials Science and Engineering C 19 (2002) 229-231.

[22]E. Kayahan, N. Ceylan, K. Esmer, Applied Surface Science, 255 (2008) 2808-2812.

[23]M. Fakis, F. Zacharatos, V. Gianneta, P. Persephonis, V. Giannetas, A. G. Nassiopoulou. Materials Science and Engineering: B, 165 (2009) 252-255.

[24]K W Li, Jiangsu University, 2013.

[25]D. Kovalev, H. Heckler, G. Polisski, J. Diener, F. Koch, physica status solidi (b) 215 (1999) 871-932.

[26]V. Petrova-Koch, T. Muschik, D. I. Kovalev, F. Koch, V. Lehmann, Materials Research Society Symposium Proceedings 283 (1993) 179-184.

[27]S. M. Prokes, Applied Physics Letters 62 (1993) 3244-3246. 